\title{
Tunable Polarization Properties of Hybrid-Guiding Liquid-Crystal Photonic Crystal Fibers
}

\author{
Dimitrios C. Zografopoulos, Student Member, IEEE, and Emmanouil E. Kriezis, Member, IEEE
}

\begin{abstract}
Tunable polarization properties in hybrid-guiding photonic crystal fibers infiltrated with nematic liquid-crystalline materials are theoretically investigated, both in the case of uniform switching of the liquid crystal molecules and for realistic nematic director patterns. It is demonstrated that the bandgap formation mechanism and the modal transmission properties in such fibers offer the possibility for electrically switchable, wavelength-selective single-polarization and/or zero to extremely high-birefringence guidance.
\end{abstract}

Index Terms-Highly birefringent fibers, nematic liquid crystals, photonic crystal fibers (PCFs), single-polarization fibers.

\section{INTRODUCTION}

$\mathbf{P}$ HOTONIC crystal fibers (PCFs) constitute a special type of optical fibers, which are characterized by the presence of a periodic cladding of cylindrical air-holes that extends around a defect core [1], [2]. Light guidance in PCFs may be achieved via two distinct physical mechanisms, that is index-guiding in a high-index glass core, or bandgap-guiding in a low-index defect core of the periodic cladding. PCFs have been under intense scientific research during the last decade as they offer extensive design capabilities, such as the elaboration of precisely defined air-hole patterns in the fiber's cladding, the utilization of different materials, or the infiltration of the cladding's capillaries with polymers, isotropic liquids, or nematic liquid crystalline materials. These possibilities allow for the sophisticated tailoring of the fiber's properties; various PCFs have been demonstrated that exhibit remarkable characteristics, such as ultraflattened dispersion [3], large mode area [4], desirable polarization properties [5], [6], or enhanced nonlinear performance [7].

Nematic liquid-crystals (NLCs) are inherently anisotropic materials consisting of elongated molecules whose axis coincides with the local optical axis. Their response to external fields or thermal variations makes them a promising candidate for the elaboration of PCFs with tunable polarization properties that may not be achieved by conventional optical fibers; indeed, many studies so far have demonstrated that the infiltration of PCFs with NLCs may boost them with special transmission and polarization properties, such as tuning of the fiber transmission

Manuscript received April 23, 2008; revised June 18, 2008. Current version published nulldate. This work was supported by the Greek General Secretariat of Research and Technology under Grant PENED/03ED936.

The authors are with the Department of Electrical and Computer Engineering, Aristotle University of Thessaloniki, Thessaloniki GR-54124, Greece (e-mail: dzogra@auth.gr; mkriezis@auth.gr).

Color versions of one or more of the figures in this paper are available online at http://ieeexplore.ieee.org.

Digital Object Identifier 10.1109/JLT.2008.928186 windows [8]-[13], switchable control of long-period grating structures [14], and tunable single-polarization (SP) or highly birefringent (HB) operation [15]-[20]. Among these properties, SP or HB fibers show particular interest; their utilization may be in certain cases indispensable, for instance to suppress polarization cross-talk and mode-dispersion in optical fiber telecommunications systems, to stabilize the operation of polarization-sensitive optical devices such as fiber-optic sensors and interferometers, to guarantee polarized laser emission in fiber laser systems, or even provide extra tuning capabilities which can be exploited in phenomena such as supercontinuum generation for optical metrology, sensor technology, and optical tomography applications [21], [22].

Recently, Sun and Chan [18] have shown that by proper material selection a NLC-infiltrated PCF may exhibit hybrid light guidance, i.e., the two orthogonal polarizations of the fundamental mode can propagate via index- and bandgap-guidance, respectively, leading to the formation of wavelength-selective windows of either SP or HB properties. In their study, it is assumed that the fiberglass index $n_{g}$ of a solid core PCF obtains a value between the infiltrated NLC ordinary $n_{O}$ and extraordinary $n_{e}$ indexes $n_{o}<n_{g}<n_{e}$, and that the NLC molecules are perfectly aligned parallel to the $\mathrm{x}-(\mathrm{y}-)$ axis. As a consequence, $\mathrm{x}$ (y-) polarized light senses high-index inclusions in the cladding $\left(n_{e}>n_{g}\right)$ and it is bandgap-guided in the core, while $\mathrm{y}-(\mathrm{x}-)$ polarized light senses low-index ones $\left(n_{o}<n_{g}\right)$ and is therefore index-guided.

In this paper, we extend the study of hybrid-guidance in NLC-infiltrated PCFs and we investigate the dispersive and polarization fiber properties related to a full electrically induced transition between a rest (zero-field, NLC molecules lying along the fiber axis) and a switched state (strong-field, NLC molecules lying along the $\mathrm{x}$ or $\mathrm{y}$ axis). We consider both the case of molecules uniformly aligned at a constant tilt angle with respect to the fiber axis and also for realistic nematic director patterns describing a Freederickz transition in cylindrical capillaries. It is demonstrated that such fibers offer the possibility for electrically tunable transitions among SP and HB states, while they exhibit a series of advantages that have not been concurrently demonstrated in other types of NLC-infiltrated PCFs proposed thus far, namely: 1) light is guided in a solid glass core and thus no material scattering/absorption loss or dispersion issues owing to the presence of the NLC are raised; 2) there is no need for selective infiltration of the cladding's capillaries; 3) no specific anchoring conditions and therefore surface treatment of the capillaries' walls is required; 4) the NLC molecules need not be fully switched in order to induce SP or HB properties; even relatively low levels of switching are sufficient; and 5) the dependence of polarization properties on 


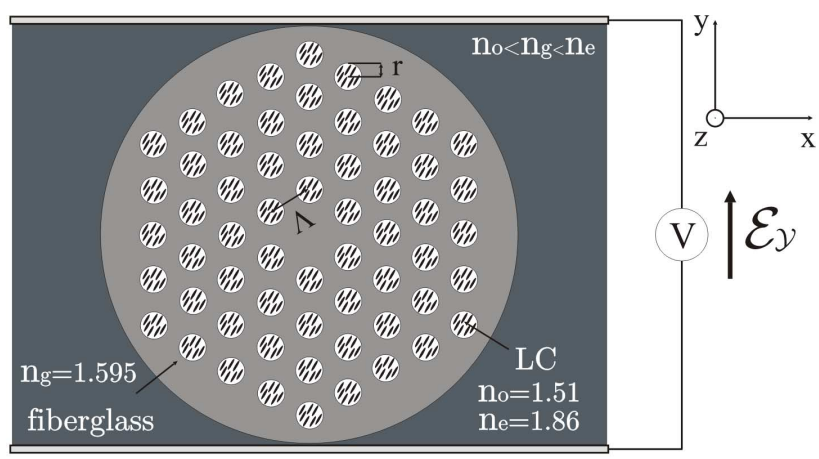

(a)
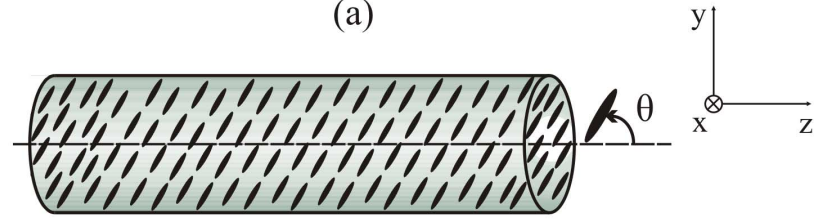

(b)

Fig. 1. (a) Structural and material parameters of the proposed hybrid-guiding photonic crystal fiber: lattice pitch $\Lambda$, lattice hole radius $r=0.25 \Lambda$, fiberglass index $n_{g}=1.595$ and infiltrated NLC ordinary and extraordinary indices, $n_{o}=1.51$ and $n_{e}=1.86$, respectively; a uniform electric field $\mathcal{E}_{y}$ is applied parallel to the y axis. (b) Definition of the tilt angle of the NLC molecules inside the infiltrated cladding capillaries.

both the applied field and the operating wavelength provides extensive control capabilities, which might be particularly exploited in novel switching or polarizing devices.

\section{TunAble Light Guidance Properties IN HYBRID-GUIDING PHOTONIC CRYSTAL FIBERS}

\section{A. Structural Parameters and Layout}

The hybrid-guiding infiltrated PCF under study follows the general layout demonstrated in Fig. 1(a); the solid glass core of a PCF is surrounded by three-rings of cylindrical air-holes of radius $r=0.25 \Lambda$, where $\Lambda$ is the pitch of the cladding's triangular lattice. The glass material is assumed to be the F2 fluorine-doped highly transmissive fiberglass by Schott AG [25], with a refractive index value of $n_{g}=1.595$ at $1530 \mathrm{~nm}$. The cladding capillaries are infiltrated by a highly birefringent NLC, characterized by extraordinary and ordinary indexes equal to $n_{e}=1.86$ and $n_{o}=1.51$ at $1550 \mathrm{~nm}$ and $30{ }^{\circ} \mathrm{C}$ [26]. The selection of these materials has been made so that the condition $n_{o}<n_{g}<n_{e}$ is met; any material combination that also fulfills it could be in general equally applicable. The fiber is placed between a pair of electrodes that may apply an electric field along the $y$ axis of the fiber's cross section as in Fig. 1(a). In the absence of any field, the NLC molecules are expected to align along the fiber axis ( $\mathrm{z}$ axis) [9], [11], which is the most favorable configuration since it minimizes the total NLC elastic energy [23]. When an electric field of intensity $\mathcal{E}_{y}$ is applied, the molecules are assumed to switch out of their rest position in the $\mathrm{yOz}$ plane, forming a tilt angle $\theta$ with the $\mathrm{z}$ axis [Fig. 1(b)].

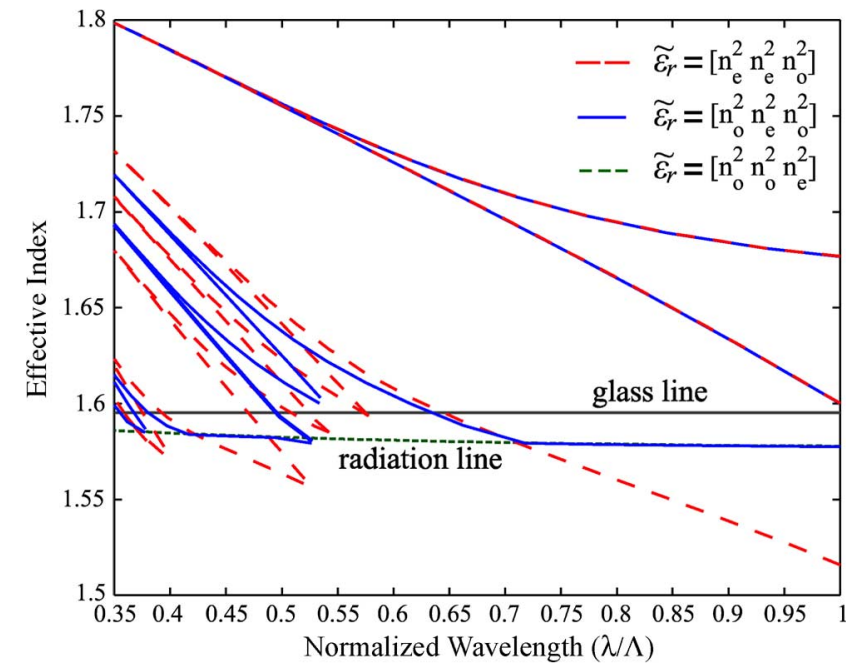

Fig. 2. Radiation line and photonic bandgaps calculated for the hybrid-guiding PCF of Fig. 1 for an infinite periodic cladding. The radiation line (dotted line) is calculated for z-aligned LC molecular director $\left(\tilde{\varepsilon}_{r}=\operatorname{diag}\left\{\mathbf{n}_{\mathrm{o}}^{2} \mathbf{n}_{\mathrm{o}}^{2} \mathbf{n}_{\mathrm{e}}^{2}\right\}\right)$, and the bandgaps for the realistic case (solid line) of y-aligned director $\left(\tilde{\varepsilon}_{r}=\operatorname{diag}\left\{\mathrm{n}_{\mathrm{o}}^{2} \mathrm{n}_{\mathrm{e}}^{2} \mathrm{n}_{\mathrm{o}}^{2}\right\}\right)$ and a reference case (dashed line) with $\tilde{\varepsilon}_{r}=\operatorname{diag}\left\{\mathrm{n}_{\mathrm{e}}^{2} \mathrm{n}_{\mathrm{e}}^{2} \mathrm{n}_{\mathrm{o}}^{2}\right\}$. The full bandgaps predicted for the realistic y-aligned director case can be approximated by the section between the radiation line and the bandgaps calculated for the reference case.

\section{B. Investigation of the Fiber's Modal Dispersive Properties for Constant Tilt Angle Values of the LC Molecules}

We begin our analysis with the study of the PCF cladding's properties, which determines the light guidance mechanism in the core, for the two extreme cases of zero- and full-switching of the NLC molecules $\left(\theta=0^{\circ}\right.$ and $\theta=90^{\circ}$, respectively). For $\theta=0^{\circ}$ all molecules align with the fiber's axis; thus, light of either x- or y-polarization senses the ordinary index $n_{o}$ in the cladding capillaries, which is lower than the matrix fiberglass index $n_{g}$. This implies that both $\mathrm{x}$ - and y-polarized light is index-guided and, taking into account the symmetry of the structure, that the fundamental PCF mode is doubly degenerate. The modal birefringence, defined as the different between the effective indexes of the $\mathrm{x}$ - and $\mathrm{y}$-polarized fundamental mode, is zero. The dispersion curve of the fundamental mode is restricted to lie between the core-line (glass line) and the fundamental space-filling mode dispersion curve (radiation line) supported by the triangular cladding. Fig. 2 shows the radiation line (dotted line) calculated by the planewave expansion method [24] for an infinite triangular cladding with $r=0.25 \Lambda$, and a dielectric tensor $\tilde{\varepsilon}_{r}=\operatorname{diag}\left\{\mathrm{n}_{\mathrm{o}}^{2} \mathrm{n}_{\mathrm{o}}^{2} \mathrm{n}_{\mathrm{e}}^{2}\right\}$ for the anisotropic NLC-infiltrated capillaries. Calculations are performed in the normalized wavelength window $0.35 \leq \lambda / \Lambda \leq 1$, which is most commonly used for practical applications. In the case of fully switched y-aligned molecules $\left(\theta=90^{\circ}\right)$, x-polarized light continues to sense low-index capillaries and it remains index-guided. On the contrary, y-polarized light senses now high-index $\left(n_{e}>n_{g}\right)$ capillaries, which allow for the formation of photonic bandgaps. Fig. 2 shows the photonic bandgaps of the infinite periodic cladding calculated by the planewave expansion method for $\tilde{\varepsilon}_{r}=\operatorname{diag}\left\{\mathrm{n}_{\mathrm{o}}^{2} \mathrm{n}_{\mathrm{e}}^{2} \mathrm{n}_{\mathrm{o}}^{2}\right\}$ and a reference case assuming $\tilde{\varepsilon}_{r}=\operatorname{diag}\left\{\mathrm{n}_{\mathrm{e}}^{2} \mathrm{n}_{\mathrm{e}}^{2} \mathrm{n}_{\mathrm{o}}^{2}\right\}$. In the first case, the 


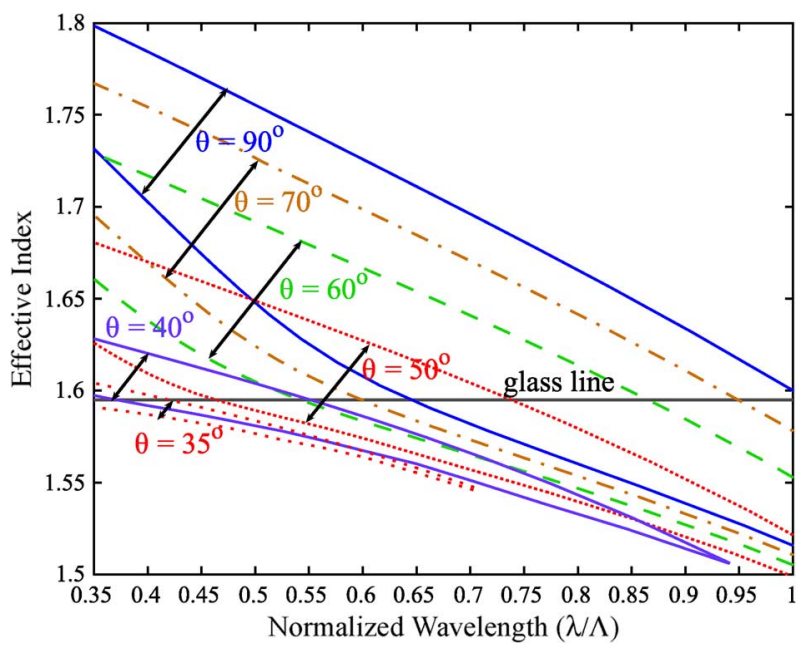

Fig. 3. Impact of the LC molecule tilt angle $\theta$ on the formation of the first-order (lowest-frequency) photonic bandgap of the hybrid-guiding PCF of Fig. 1 with respect to the bandgap-guided y-polarized guided modes. The bandgap window below the glass line, which may support bandgap modes, begins to emerge for $\theta=35^{\circ}$ and progressively broadens and shifts to longer wavelengths as $\theta \rightarrow$ $90^{\circ}$.

realistic structure (y-aligned molecules) is simulated; nevertheless the resulting bandgaps (solid lines) are truncated below the glass line by the radiation line that corresponds to the x-polarized modes. Thus, full information cannot be retrieved for the bandgap limits that apply only to the y-polarized modes. The full photonic bandgap edges for the y-polarized mode can be assessed by considering the second reference case; below the fiberglass line the first-order (lowest frequency) bandgap shows almost perfect agreement between the realistic and the reference case, while the second-order bandgap below the glass line can still be approximated by the reference case. From a theoretical point of view, Fig. 2 demonstrates how the hybrid behaviour of the fiber's cladding for $\theta=90^{\circ}$ may be considered to derive from two seperate phenomena corresponding to the low- and high-index inclusions sensed by index-guided $\mathrm{x}$ and bandgap-guided y-polarized light, respectively.

Fig. 3 shows the impact of the tilt angle $\theta$ on the formation of the first-order bandgap corresponding to y-polarized light. Starting for $\theta=90^{\circ}$, as $\theta$ obtains lower values the effective capillary index sensed by y-polarized light progressively drops, leading to the shrinkage of the bandgap and its shiftage to higher frequencies. For $\theta=35^{\circ}$ only a small portion of the bandgap extends below the fiberglass line, while for $\theta=30^{\circ}$ no bandgap was predicted below the glass line for $0.35<\lambda / \Lambda<1$, indicating that no bandgap-guidance is supported for $\theta \leq 30^{\circ}$.

The analysis of the modal dispersive properties for the finite three-ring cladding hybrid-guiding PCF have been calculated by a proper variation of the compact finite-difference time-domain (C-FTDT) method, which is capable of dealing with waveguiding problems where anisotropic materials are involved [27]. Fig. 4 shows the dispersion curves for the index-guided $\mathrm{x}$ - and $y$-polarized fundamental mode of the PCF under study for a tilt angle of $\theta=0^{\circ}$ and $\theta=20^{\circ}$ related to the LC molecule alignment. The radiation line has been calculated in both cases for $\theta=0^{\circ}$ and refers to the finite three-ring cladding structure of Fig. 1. For $\theta=0^{\circ}$ (rest/zero-field case) all molecules lie

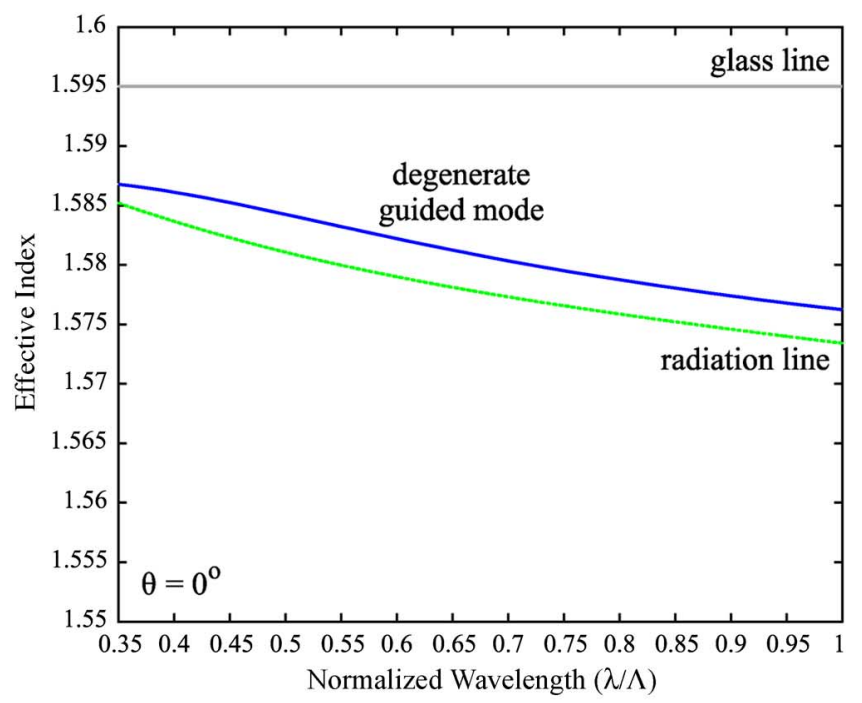

(a)

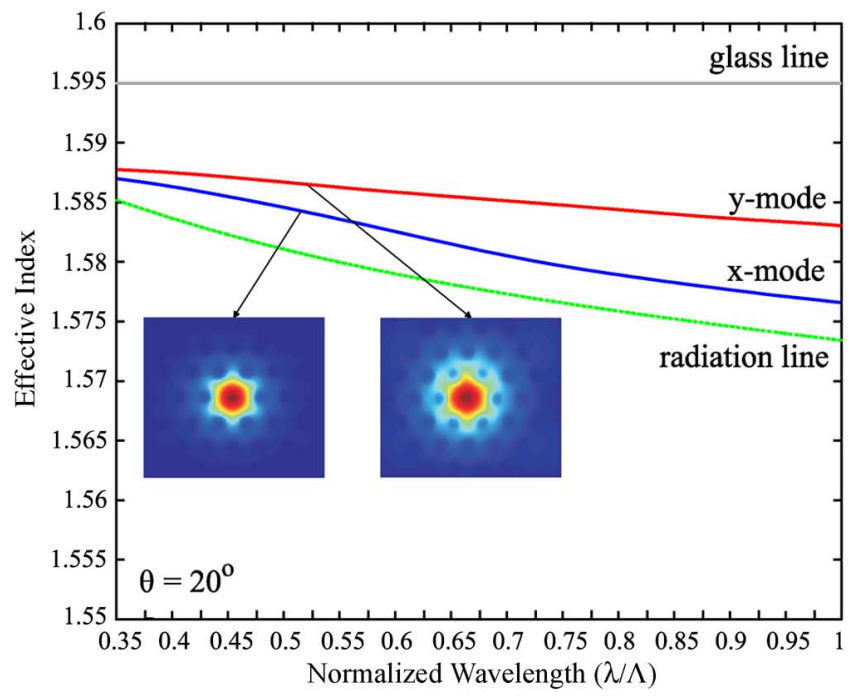

(b)

Fig. 4. Dispersion curves for the index-guided $\mathrm{x}$ - and $\mathrm{y}$-polarized fundamental mode of the hybrid-guiding PCF under study for a LC molecule tilt angle of (a) $\theta=0^{\circ}$ and (b) $\theta=20^{\circ}$. In the case of $\theta=0^{\circ}$ all molecules lie parallel to the fiber axis and the fundamental mode is degenerate; as $\theta$ obtains higher values the $\mathrm{x}$-polarized mode dispersion curve remains unaffected, while the y-polarized one rises towards the glass line, leading to high values of modal birefringence. Insets in (b) show the electric field modal profile at $\lambda / \Lambda=0.51$; the $\mathrm{x}$-polarized mode shows better confinement as it senses the ordinary index $n_{o}$ in the infiltrated capillaries, contrary to the y-polarized one that senses a higher effective index.

parallel to the fiber axis, the fundamental mode is degenerate and consequently modal birefringence is zero. As the NLC material progressively switches and $\theta$ obtains higher values, the $\mathrm{x}$-polarized mode dispersion curve remains almost unaffected, while the y-polarized one rises towards the glass line, leading to high values of modal birefringence. This is interpreted as follows: as the NLC molecules tilt in the y-z plane, x-polarized light is always perpendicularly polarized to the nematic director (sensing the ordinary index $n_{o}$ in the capillaries), while the effective index value sensed by y-polarized light rises with $\theta$. The insets in Fig. 4(b) show two electric field modal profiles at $\lambda / \Lambda=0.51$, corresponding to the $\mathrm{x}$ - and $\mathrm{y}$-polarized 
fundamental mode. The x-polarized mode exhibits better confinement as it senses a higher contrast in the low-index cladding $\left(n_{g} \leftrightarrow n_{o}\right)$, contrary to the y-polarized one that senses a higher effective index in the infiltrated capillaries.

As the NLC molecules are further switched, the fiber enters a single-polarization state (around $\theta=30^{\circ}$ ), where only the $\mathrm{x}$-polarized fundamental mode is allowed to propagate. This is because the effective index sensed by y-polarized light becomes too close or higher than $n_{g}$, prohibiting thus index-guiding, while at the same time it is not sufficiently high to lead to the emergence of photonic bandgaps below the fiberglass line, as discussed in the context of Fig. 3. The tilt angle threshold that marks the transition to the single-polarization state cannot be univocally determined; it is related to the confinement losses of the y-polarized mode that rise significantly as its effective index approaches $n_{g}$ and lead to high values of polarization dependent losses, which in general depend on the material parameters, the hole-radius and the number of rings in the cladding. Higher values of $\theta$ are needed in order to induce hybrid-guidance; Fig. 5 shows the dispersion curves for both the index-guided $\mathrm{x}$-polarized and the bandgap-guided $\mathrm{y}$-polarized fundamental mode for a LC molecule tilt angle of $\theta=60^{\circ}$ and $\theta=90^{\circ}$. It can be noticed that the PCF now allows for the propagation of $y$-polarized light within the wavelength windows related to the photonic bandgaps supported by the cladding for each specific value of $\theta$ [confinement is induced by the bandgap effect as demonstrated by the modal profile insets in Fig. 5(b)]. Thus, high values of modal birefringence are predicted within the bandgap windows, while single-polarization operation occurs in the rest of the wavelength window under study.

The overall dispersive behaviour of the hybrid-guiding PCF under study is summarized in Fig. 6, which shows the modal birefringence curves for the complete NLC-molecule switching transition from $\theta=0^{\circ}$ to $\theta=90^{\circ}$, with a step of $10^{\circ}$. For $\theta \leq 20^{\circ}$ the fiber operates in a HB state in the whole normalized wavelength window $0.35<\lambda / \Lambda<1$, with birefringence values of up to $-6 \times 10^{-3}$. For $\theta=30^{\circ}$ birefringence cannot be defined as the fiber is in a SP state (propagation of the y-polarized mode is not supported at all). For $\theta \geq 40^{\circ}$ the y-polarized mode may be guided within the photonic bandgaps extending below the glass line, which also define the limits for HB operation. The exact position of the HB wavelength windows may be adjusted by controlling the tilt angle $\theta$. Extremely high values of modal birefringence are predicted for guidance in the first-order bandgap, up to more than $5 \times 10^{-2}$. It is worth mentioning that these values are predicted for moderate switching values $\left(\theta=50^{\circ}\right)$, while it is demonstrated that further switching, which in turn requires the application of higher voltage, does not yield any specific advantages. Even for $\theta=40^{\circ}$ there is a well defined extra HB window for $0.55<\lambda / \Lambda<0.75$, outside of which the fiber operates in a SP state. Most interestingly, Fig. 6 indicates that for a given operation wavelength, the fiber polarization state may be tuned progressively from zero to high values of modal birefringence $\left(10^{-3}\right.$ to $\left.10^{-2}\right)$, followed by single-polarization, and finally by extremely highly birefringence $\left(>10^{-2}\right)$ operation. The single-polarization and highly birefringence states appear for different switching angle values with respect to the normalized wavelength; the selec-

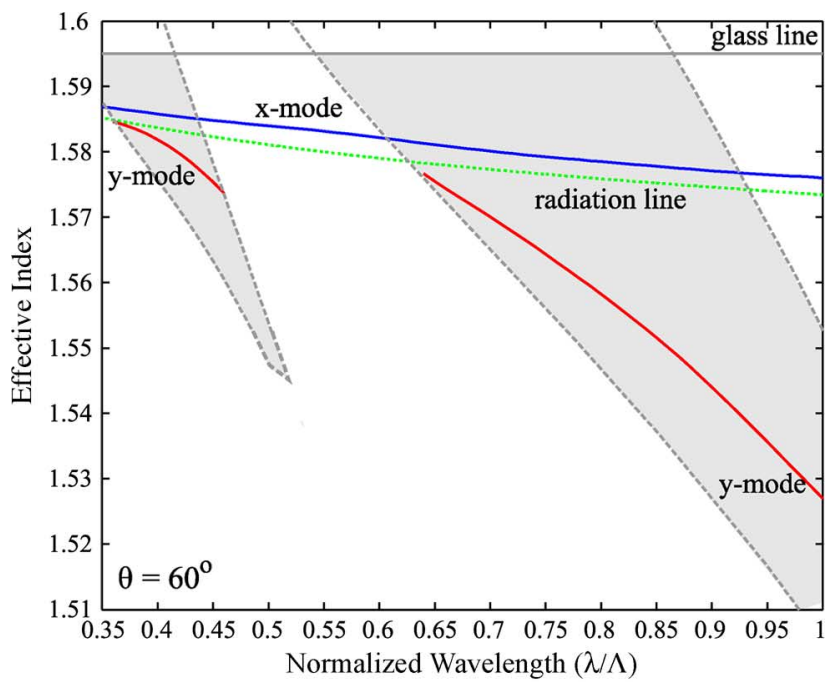

(a)

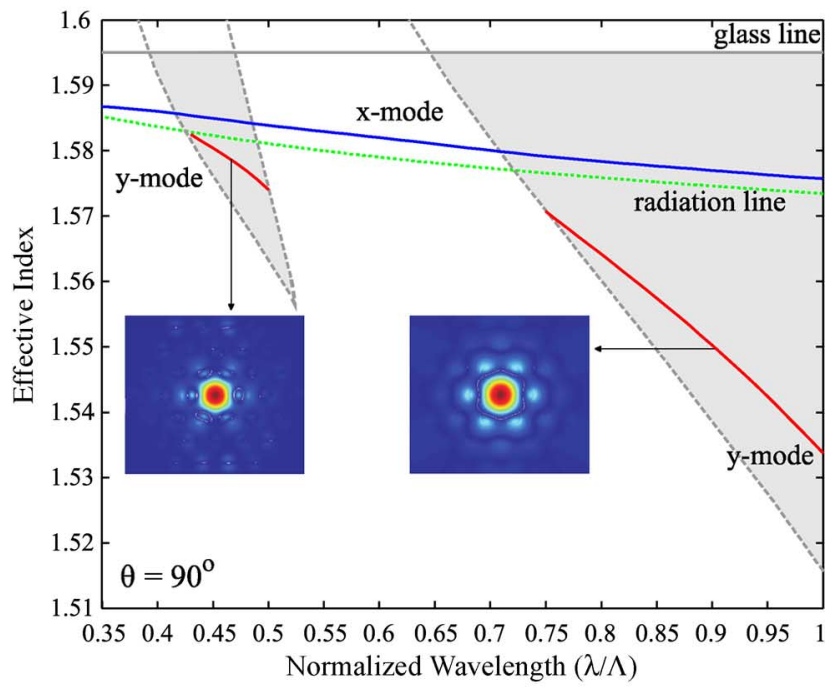

(b)

Fig. 5. Dispersion curves for the index-guided x-polarized and the bandgapguided y-polarized fundamental mode of the hybrid-guiding PCF under study for a LC molecule tilt angle of (a) $\theta=60^{\circ}$ and (b) $\theta=90^{\circ}$. Insets in (b) show the electric field modal profile of the y-polarize mode at $\lambda / \Lambda=0.46$ (secondorder bandgap) and at $\lambda / \Lambda=0.9$ (first-order bandgap).

tion of the normalized operation wavelength and therefore the lattice pitch depends on the required degree of tunability and birefringence values. Referring to Fig. 6, the normalized wavelength window $0.7<\lambda / \Lambda<0.8$ offers both the possibility to obtain high values $(>0.02)$ of birefringence for low switching angles and extensive tunability as $\theta$ rises from $40^{\circ}$ to $90^{\circ}$. If higher values $(>0.04)$ of birefringence are targeted the window $0.9<\lambda / \Lambda<0.1$ is more appropriate, although tunability is somehow restricted in the interval $50^{\circ}<\theta<90^{\circ}$.

\section{Investigation of Fiber's Modal Dispersive Properties for Realistic LC Nematic Director Patterns}

Although the description of the NLC-molecule switching via the constant tilt angle $\theta$ can be considered indicative of the transition from the rest to the switched state, it cannot be necessarily expected to achieve in practice. In order to predict the realistic behavior and performance of NLC infiltrated capillaries, 


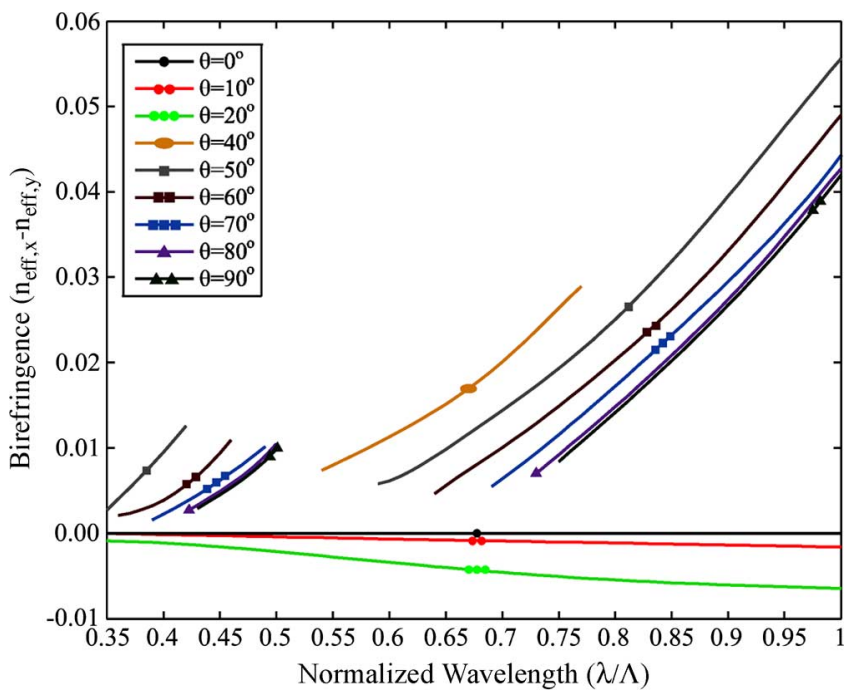

Fig. 6. Family of birefringence curves for various values of the LC molecule tilt angle $\theta$. For $\theta=0^{\circ}$ the fundamental guided mode is degenerate (zero birefringence), for $\theta=10^{\circ}, 20^{\circ}$ both polarizations are index-guided with $n_{\text {eff }, \mathrm{y}}>n_{\text {eff }, \mathrm{x}}$, while for $\theta \geq 40^{\circ}$ the y-polarized mode is guided only inside the corresponding photonic bandgaps with $n_{\text {eff, }}>n_{\text {eff,y }}$. Very high levels of modal birefringence $\left(>2 \times 10^{-2}\right)$ are predicted even for relatively low switching angles $\left(\theta=40^{\circ}\right)$.

it is required to calculate the response of the nematic material to the application of the external electric field. The exact configuration of the nematic director in a confined geometry can be resolved via the minimization of the Oseen-Frank free elastic energy over the material's bulk, and the solution of the resultant Euler-Lagrange differential equation [28], [29]. Our calculations were performed under the assumption of a uniform y-aligned electric field, as the electric field lines are not expected to deviate from the y axis more than a few degrees since the dielectric materials involved (fiberglass and nematic liquid crystal) have close values of electric permittivity [10]. In addition, the one-elastic constant approximation and hard homogeneous anchoring conditions $\left(\theta=0^{\circ}\right)$ at the capillaries' walls were assumed. The material parameters used were that of the considered highly birefringent nematic material, namely $\Delta \varepsilon=16.6$, and $K=0.5\left(K_{11}+K_{33}\right)=22.8 \mathrm{pN}$ [26].

Fig. 7 shows the average tilt angle $\bar{\theta}$ calculated over the cross section of a LC-infiltrated capillary with respect to the applied voltage drop for homogeneous anchoring conditions (molecules lying parallel to the cavity's walls). The switching of the LC molecules exhibits a Freederickz-like transition; it begins to occur above a voltage-threshold $V_{\text {th }} \simeq 1.9 \mathrm{~V}$, it is intensely enhanced for $2 \leq V \leq 10 \mathrm{~V}$, while for higher voltage values $\bar{\theta}$ slowly converges towards an upper limit. It is pointed out that the characteristics of the transition depend only on the applied voltage, that is the voltage drop across the capillary's diameter, and not on the physical dimensions of the capillary; the intensity of the applied field needed to induce a given voltage drop may be approximated by $\mathcal{E} \simeq V / 2 r$.

The fiber modal analysis described in Section II-B has been repeated by inserting the realistic nematic director profiles (such as the insets shown in Fig. 7), calculated by the finite-element method, into the C-FDTD code. Fig. 8 shows a comparison of

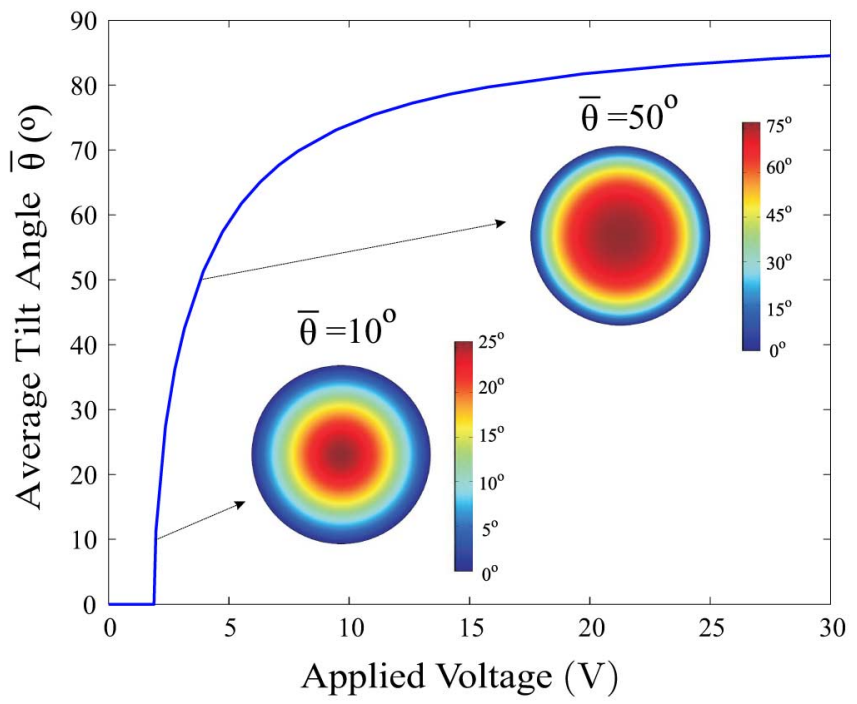

Fig. 7. Average tilt angle $\bar{\theta}$ in a NLC-infiltrated capillary with respect to the applied voltage drop for homogeneous anchoring conditions. The switching of the NLC molecules begins to occur above a voltage-threshold $V_{\mathrm{th}} \simeq 1.9 \mathrm{~V}$, it is intensely enhanced for $2 \leq V \leq 10 \mathrm{~V}$, while for higher voltage values $\bar{\theta}$ slowly converges towards an upper limit. Insets show the local tilt angle configuration in the capillary for two values of applied voltage $V=2 \mathrm{~V}$ and $V=4 \mathrm{~V}$.

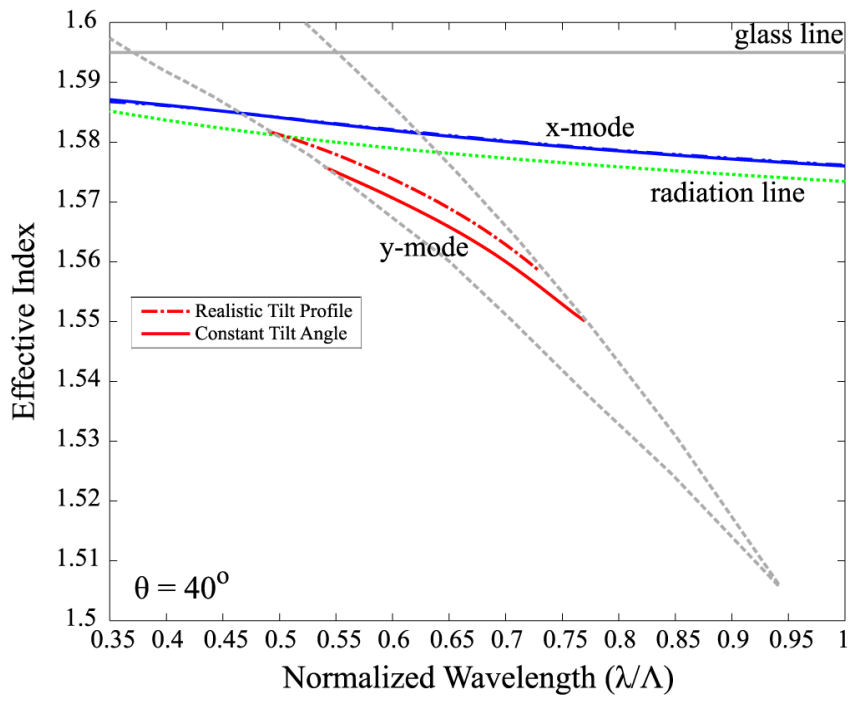

Fig. 8. Dispersion curves for the index-guided x-polarized and the bandgapguided y-polarized fundamental mode of the hybrid-guiding PCF under study calculated for a constant NLC molecule tilt angle of $\theta=40^{\circ}$ and a realistic nematic director profile of equal average tilt angle $\left(\bar{\theta}=40^{\circ}\right)$. The y-polarized mode is in both cases bandgap-guided, albeit following different dispersion curves, while the dispersive properties of the $\mathrm{x}$-polarized mode remain practically identical.

the results corresponding to both the constant tilt angle approximation and a corresponding realistic profile, selected so that $\theta=\bar{\theta}=40^{\circ}$. As far as the x-polarized mode is concerned, the modal dispersion curve for both cases is practically identical; the dispersion curve of the bandgap y-polarized mode is shifted, leading to a shiftage of the HB window as well. Nevertheless, the basic properties of the fiber's hybrid-guiding mechanism are similar in both cases, the only difference being that SP and HB operation is not demonstrated in the same wavelength windows 


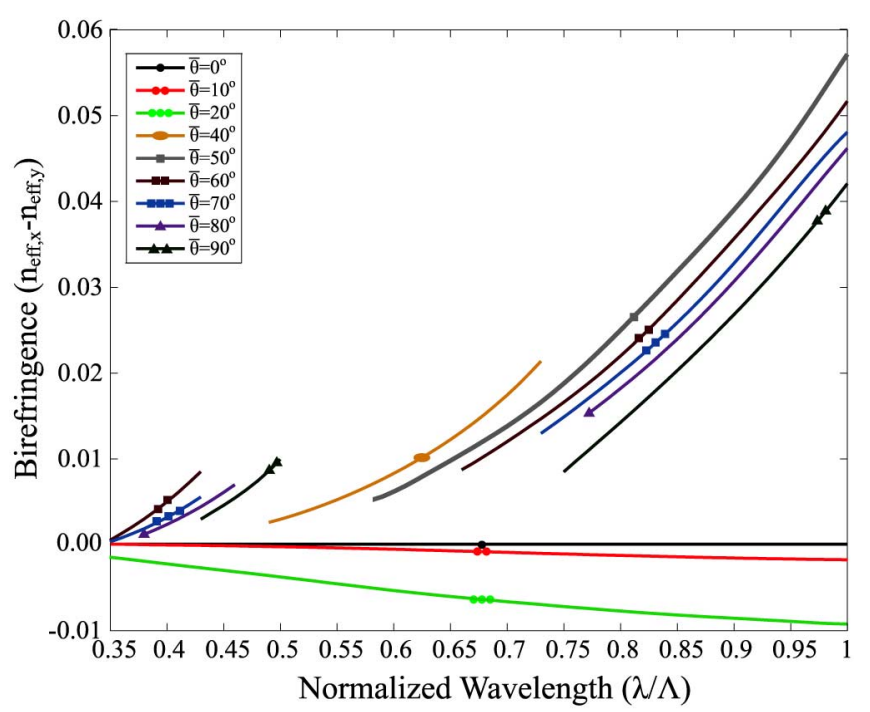

Fig. 9. Family of birefringence curves for various realistic nematic director patterns characterized by the average value of the LC molecule tilt angle $\bar{\theta}$. As in the case of the birefringence curves calculated for constant tilt angles (Fig. 6), for $\theta=10^{\circ}, 20^{\circ}$ both polarizations are index-guided with $n_{\text {eff }, \mathrm{x}}<n_{\text {eff, } \mathrm{y}}$, while for $\theta \geq 40^{\circ}$ the y-polarized mode is guided only inside the corresponding photonic bandgaps with $n_{\mathrm{eff}, \mathrm{x}}>n_{\text {eff, } \mathrm{y}}$. Very high levels of modal birefringence $\left(>2 \times 10^{-2}\right)$ are again predicted for relatively low switching angles $\left(\theta=40^{\circ}\right)$.

for profiles corresponding to constant values of $\theta$ and to realistic nematic director configurations. This behaviour is justified since although these profiles express a similar average orientation of the NLC molecules, they are to a certain extent diversified and they cannot be expected to lead to exact dispersive modal properties.

Fig. 9 shows the family of modal birefringence curves for the hybrid-guiding PCF under study, for parameters directly comparable to those of Fig. 6, the only difference being that these curves are calculated for the realistic director profiles, with the particular average tilt angle $\bar{\theta}$. All salient features observed in the constant tilt angle analysis of Fig. 6 may also be distinguished in this case as well. Modal birefringence obtains values up to $-9 \times 10^{-3}$ for $\bar{\theta}=20^{\circ}$, a SP operation state follows and finally wavelength-selective $\mathrm{HB}$ is predicted for $\bar{\theta} \geq 40^{\circ}$ with extremely high values of $\left(>10^{-2}\right)$ birefringence. For $\bar{\theta}=40^{\circ}$, which is accomplished for a voltage drop of less than $10 \mathrm{~V}$ across each capillary (Fig. 7), the fiber already operates in wavelength-selective extremely HB state. The two cases are diversified mainly in terms of the positions of the photonic bandgaps and consequently the HB operation windows, which are somehow shifted towards higher frequencies in the case of the realistic nematic director patterns.

\section{CONCLUSION}

Electrically switchable single-polarization and highly birefringent hybrid-guiding photonic crystal fibers can be designed by infiltrating the cladding's holes of a conventional index-guiding photonic crystal fiber with a nematic liquid crystalline material. Various distinct operation states are predicted, namely zero-, high-, or extremely high birefringence, and single polarization guidance. Wavelength-selective transition among these states may be substantially tuned by properly adjusting the voltage of the applied electric field. The above desirable properties come at no cost with respect to NLC material losses/dispersion, the need for specific surface treatment or selective infiltration of the infiltrated capillaries, or the application of extremely high electric field intensity. The polarization features of such fibers may find significant utilization in applications where extensive light polarization control or switching is indispensable.

\section{REFERENCES}

[1] A. Bjarklev, J. Broeng, and A. S. Bjarklev, Photonic Crystal Fibers. Norwell, MA: Kluwer, 2003.

[2] J. C. Knight, "Photonic crystal fibers," Nature, vol. 424, pp. 847-851, 2003.

[3] W. H. Reeves, J. C. Knight, P. St. Russell, and P. J. Roberts, "Demonstration of ultra-flattened dispersion in photonic crystal fibers," Opt. Exp., vol. 10, pp. 609-613, 2002.

[4] J. C. Knight, T. A. Birks, R. F. Cregan, P. St. Russell, and J. P. de Sandro, "Large mode area photonic crystal fibre," Electron. Lett., vol. 34, pp. 1347-1348, 1998.

[5] J. Ju, W. Jin, and M. S. Demokan, "Design of single-polarization single-mode photonic crystal fiber at 1.30 and $1.55 \mu \mathrm{m}$, , J. Lightw. Technol., vol. 24, no. 2, pp. 825-830, Feb. 2006.

[6] C. Kerbage and B. J. Eggleton, "Numerical analysis and experimental design of tunable biregringence in microstructured optical fiber," Opt. Exp., vol. 10, pp. 245-255, 2002.

[7] K. P. Hansen, "Dispersion flattened hybrid-core nonlinear photonic crystal fiber," Opt. Exp., vol. 11, pp. 1503-1509, 2003.

[8] T. T. Larsen, A. Bjarklev, D. S. Hermann, and J. Broeng, "Optical devices based on liquid crystal photonic fibers," Opt. Exp., vol. 11, pp. 2589-2596, 2003.

[9] T. T. Alkeskjold, J. Lægsgaard, A. Bjarklev, D. S. Hermann, J. Broeng, J. Li, and S. T. Wu, "All-optical modulation in dye-doped nematic liquid crystal photonic bandgap fibers," Opt. Exp., vol. 12, pp. 5857-5871, 2004.

[10] L. Scolari, T. T. Alkeskjold, J. Riishede, and A. Bjarklev, "Continuously tunable devices based on electrical control of dual-frequency liquid crystal filled photonic bandgap fibers," Opt. Exp., vol. 13, pp. 7483-7496, 2005.

[11] M. W. Haakestad, T. T. Alkeskjold, M. Nielsen, L. Scolari, J. Riishede, H. E. Engan, and A. Bjarklev, "Electrically tunable photonic bandgap guidance in a liquid-crystal-filled photonic crystal fiber," IEEE Photon. Technol. Lett., vol. 17, no. 4, pp. 819-821, Apr. 2005.

[12] T. R. Woliński, K. Szaniawska, S. Ertman, P. Lesiak, A. W. Domański, R. Dạbrowski, E. Nowinowski-Kruszelnicki, and J. Wójcik, "Influence of temperature and electrical fields on propagation properties of photonic liquid-crystal fibers," Meas. Sci. Technol., vol. 17, pp. 985-991, 2006.

[13] T. R. Woliński, S. Ertman, P. Lesiak, A. W. Domański, A. Czapla, R. Dạbrowski, E. Nowinowski-Kruszelnicki, and J. Wójcik, "Photonic liquid crystal fibers-A new challenge for fiber optics and liquid crystals photonics," Opto-Electron. Rev., vol. 14, pp. 329-334, 2006.

[14] D. Noordegraaf, L. Scolari, J. Lægsgaard, L. Rindorf, and T. T. Alkeskjold, "Electrically and mechanically induced long period gratings in liquid crystal photonic bandgap fibers," Opt. Exp., vol. 15, pp. 7901-7912, 2007.

[15] T. T. Alkeskjold and A. Bjarklev, "Electrically controlled broadband liquid crystal photonic bandgap fiber polarimeter," Opt. Lett., vol. 32, pp. 1707-1709, 2007.

[16] G. Tartarini, M. Pansera, T. T. Alkeskjold, A. Bjarklev, and P. Bassi, "Polarization properties of elliptical-hole liquid crystal photonic bandgap fibers," J. Lightw. Technol., vol. 25, no. 9, pp. 2522-2530, Sep. 2007.

[17] J. Sun and C. C. Chan, "Effect of liquid crystal alignment on bandgap formation in photonic bandgap fibers," Opt. Lett., vol. 32, pp. 1989-1991, 2007.

[18] J. Sun and C. C. Chan, "Hybrid guiding in liquid-crystal photonic crystal fibers," J. Opt. Soc. Amer. B, vol. 24, pp. 2640-2646, 2007.

[19] D. C. Zografopoulos, E. E. Kriezis, and T. D. Tsiboukis, "Photonic crystal-liquid crystal fibers for single-polarization or high-birefringence guidance," Opt. Exp., vol. 14, pp. 914-925, 2006.

[20] D. C. Zografopoulos, E. E. Kriezis, and T. D. Tsiboukis, "Tunable highly birefringent bandgap-guiding liquid-crystal microstructured fibers," J. Lightw. Technol., vol. 24, no. 9, pp. 3427-3432, Sep. 2006. 
[21] H. Lim, A. Chong, and F. Wise, "Environmentally-stable femtosecond ytterbium fiber laser with birefringent photonic bandgap fiber," Opt. Exp., vol. 13, pp. 3460-3464, 2005.

[22] M. Lehtonen, G. Genty, H. Ludvigsen, and M. Kaivola, "Supercontinuum generation in a highly birefringent microstructured fiber," App. Phys. Lett., vol. 82, pp. 2197-2199, 2003.

[23] S. V. Burylov, "Equilibrium configuration of a nematic liquid crystal confined to a cylindrical cavity," J. Exp. Theor. Phys., vol. 85, pp. 873-886, 1997.

[24] S. G. Johnson and J. D. Joannopoulos, "Block-iterative frequency-domain methods for maxwell's equations in a planewave basis," Opt. Express, vol. 8, pp. 173-190, 2001.

[25] SCHOTT AG Fluorine-Doped Optical Fiberglass Series [Online]. Available: http://www.schott.com

[26] S. Gauza, J. Li, S.-T. Wu, A. Spadło, R. Dạbrowski, Y.-N. Tzeng, and K.-L. Cheng, "High birefringence and high resistivity isothiocyanate-based nematic liquid crystal mixtures," Liq. Cryst, vol. 32, pp. 1077-1085, 2005.

[27] A. P. Zhao, J. Juntunen, and A. V. Räisänen, “A generalized compact 2-d fdtd model for the analysis of guided modes of anisotropic waveguides with arbitrary tensor permittivity," Microw. Opt. Tech. Lett., vol. 18, pp. 17-23, 1998.

[28] F. A. Fernández, S. E. Day, P. Trwoga, H. F. Deng, and R. James, "Three-dimensional modelling of liquid crystal display cells using finite elements," Mol. Cryst. Liq. Cryst., vol. 375, pp. 291-299, 2002.

[29] I. W. Stewart, The Static and Dynamic Continuum Theory of Liquid Crystals. New York: Taylor and Francis, 2004.

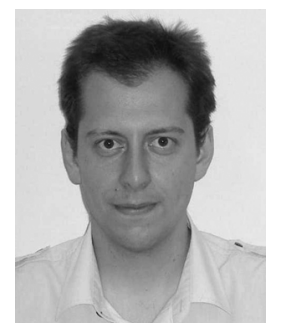

Dimitrios C. Zografopoulos (S'08) was born in Thessaloniki, Greece, in 1980. He received the Diploma degree in electrical and computer engineering from the Aristotle University of Thessaloniki (AUTH), Greece, in 2003, where he is currently working toward the Ph.D. degree.

His research interests include computational electromagnetics, emphasizing on the analysis and design of optical waveguides and photonic/liquid crystal devices.

Mr. Zografopoulos is a member of the IEEE Lasers and Electro-Optics Society.

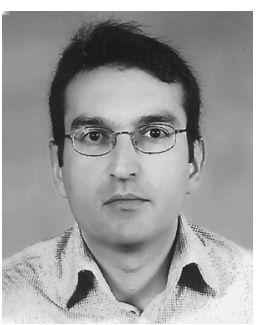

Emmanouil E. Kriezis (S'92-M'96) was born in Thessaloniki, Greece, in 1968. He received the Diploma degree in electrical engineering and the Doctorate degree in electrical engineering, both from the Department of Electrical and Computer Engineering, Aristotle University of Thessaloniki (AUTH), in 1991 and 1996, respectively. His doctoral thesis focused on the development of full vector beam propagation methods for light wave propagation in integrated optical devices.

In 1998, he joined the Department of Engineering Science, University of Oxford, U.K., initially as an EPSRC Postdoctoral Researcher. In 2001, he was awarded the prestigious Royal Society University Research Fellowship to study light propagation in complex anisotropic media. In September 2002, he was appointed Assistant Professor in Optical and Microwave Communications at the Department of Electrical and Computer Engineering, AUTH. His research interests include methods of lightwave propagation, analysis of integrated optical devices, liquid crystal devices, photonic crystals, and diffractive elements.

Dr. Kriezis is a member of the IEEE Lasers and Electro-Optics Society. 